\title{
Study of Mass and Temperature Distribution in Clump Near the Sp1 Nebula at the Galactic Latitude $+01.9^{\circ}$
}

\author{
Rajesh Shrestha*, Bibek Dhungana, Bashudev Bhandari, Rajan Dev Pant \\ Tri-Chandra Multiple Campus, Tribhuvan University, Kathmandu, Nepal \\ Email: ^rajeshshrestha402@gmail.com
}

How to cite this paper: Shrestha, R., Dhungana, B., Bhandari, B. and Pant, R.D. (2021) Study of Mass and Temperature Distribution in Clump Near the Sp1 Nebula at the Galactic Latitude $+01.9^{\circ}$. Open Access Library Journal, 8: e8052.

https://doi.org/10.4236/oalib.1108052

Received: October 6, 2021

Accepted: November 15, 2021

Published: November 18, 2021

Copyright $\odot 2021$ by author(s) and Open Access Library Inc.

This work is licensed under the Creative Commons Attribution International License (CC BY 4.0).

http://creativecommons.org/licenses/by/4.0/

\begin{abstract}
The study of the dust structure around a clump of star forming region sp1 has been performed at $+01.9^{\circ}$ latitude in the Infrared Science Archive (IRAS) map. The two different fits images of $60 \mu \mathrm{m}$ and $100 \mu \mathrm{m}$ are downloaded from sky view virtual observatory and a clump is selected as a study region. The flux densities at different points have been calculated using Aladin software. By analyzing two different Flexible Image Transport System (FITS) images of $60 \mu \mathrm{m}$ and $100 \mu \mathrm{m}$ the average temperature is obtained about 34.43 $\mathrm{K}$. The average diameter of this clump is found to have $4.47 \mathrm{pc}$. The plot of flux densities along diameter is found to have a Gaussian curve. Mass of the clump is found $0.036185 \mathrm{M}_{\odot}$, whereas the jeans mass for that clump is about $27,896.75 \mathrm{M}_{\odot}$. Hence from those results, we find that the system is self-isolated with radiating dense core and there is no possibility of any star formation in that region.
\end{abstract}

\section{Subject Areas \\ Astrophysics, Cosmology}

\section{Keywords}

IRAS, FIT Images, Aladin Software, Jeans Mass and Star Formation

\section{Introduction}

As we understood that the interstellar cloud plays a significant role in the staller evolution. Different non-linear phenomena can occur in interstellar space due to influence of shock wave, gravitation, radiation, magnetic field etc. which is studied in idealized model [1], but unable to describe all such phenomena in single 
theory. Also, interstellar cloud is highly abundant in chemical composition, over 40 different chemical species and variety of molecules have been discovered in interstellar cloud. The advanced and complex molecules discovered in interstellar medium were the greatest surprise to astronomical community. Different factors such as chemical composition of cloud \& dust, grain size, temperature, gravitational field etc. can directly affect the state of interstellar cloud [2].

Most part of the space is almost empty only certain proportion of gas and dust is found between the stars and galaxies. This region of dust and gas is known as interstellar medium (ISM) and has constitution of Hydrogen (HI), Ionized gas (HII), Molecular gas (H2), some heavier molecules and dusts suggested by Smith H.E in 2012. Densities of ISM are highly varied from $10^{-4}$ ions $\mathrm{cm}^{3}$ in ionized region to $10^{6}$ molecules $\mathrm{cm}^{3}$ in molecular regions. If we look over the composition of ISM $99 \%$ of it is in gaseous molecules and $1 \%$ dust is particles [3].

The combined project of the US, UK and the Netherlands performed the survey of entire sky and made a complete map. The survey has carried out in 12, 25, $60 \& 100 \mu \mathrm{m}$ wavelength, detects each point source and supplies photometry data. This survey provides data of more than 250,000 point sources [4].

In this study a clump of dusts-cloud is selected bear the Sp1 nebula, which lies at $1450 \mathrm{pc}$ distance. The fits images of $60 \mu \mathrm{m}$ and $100 \mu \mathrm{m}$ were taken from IRAS map. The flux densities at different points are calculated by using ALADIN v11 Software. By observing the flux densities at $60 \mu \mathrm{m}$ and $100 \mu \mathrm{m}$ we calculate the average temperature of cloud. The major and minor diameters are also obtained by using parallax method to the data of angle between two points obtained from fits images using ALADIN v11 software.

The variation of flux densities is also calculated along the major and minor diameters. From those calculated data and with the help of some formulas adopted from previous research papers, we calculate the dust mass and jeans mass for that particular location to check whether that region is in process of star formation. In addition, the Color map according to the flux densities is obtained using Python. Sp1 is a planetary nebula, the formation of a star near it, and the study of its interstellar medium is also crucial in astronomy. This study is carried out to find out the possibilities of the formation of a star in a large clump near the Sp1 nebula at galactic latitude $+01.9^{\circ}$.

\section{Theory}

\subsection{Dust Temperature}

The dust temperature can be determined by measuring the flux densities at 60 $\mu \mathrm{m}$ and $100 \mu \mathrm{m}$ from IRSA map. The ratio of flux densities at $60 \mu \mathrm{m}$ and $100 \mu \mathrm{m}$ is used to calculate the temperature of the dust. The dust temperature $\mathrm{T}$ in each pixel of a FITS image can be obtained by assuming that the dust in a single beam is isothermal and that the observed ratio of $60 \mu \mathrm{m}$ to $100 \mu \mathrm{m}$ emission is due to blackbody radiation from dust grains at $\mathrm{T}$, modified by a power law emissivity spectral index [5]. Now, considering that dust emission is optically thin at $60 \mu \mathrm{m}$ 
and $100 \mu \mathrm{m}$, we can write the ratio of the flux densities is

$$
R=0.6^{-(3+\beta)} \frac{\mathrm{e}^{144 / T}-1}{\mathrm{e}^{240 / T}-1}
$$

Neglecting 1 from both nominator and denominator

$$
R=0.6^{-(3+\beta)} \frac{\mathrm{e}^{144 / T}}{\mathrm{e}^{240 / T}}
$$

Taking natural logarithm on both sides

$$
T=\frac{-96}{\ln \left(R \times 0.6^{(3+\beta)}\right)}
$$

Thus if the value of $\beta$ is known, the value of dust temperature will be known. In this research the value $\beta$ is assume to be $2[6]$.

\subsection{Dust Mass}

The dust mass is measured by observing the flux densities of the dust at $100 \mu \mathrm{m}$ in IRAS map. The longer wave can give more precise so we select $100 \mu \mathrm{m}$ map instead of $60 \mu \mathrm{m}$. The flux density $F_{n}$ of the cloud at distance $D$, with $N$ spherical dust grains with each of cross-section $\sigma$, temperature $T$, and the emissivity $Q_{V}$ is given by

$$
F_{v}=\frac{N \sigma Q_{v} B(v, T)}{D^{2}}
$$

This calculation gives the flux density [7].

Let $v$ be the volume of each grain then the total volume is given by

$$
V=N v
$$

Replacing the value of $N$, the volume is given by

$$
V=\frac{F D^{2} v}{\sigma Q_{v} B(v, T)}
$$

The cross-section $\sigma$ is

$$
\sigma=\pi a^{2}
$$

where a is the radius of grain, similarly the value of volume of grain is given by

$$
v=\frac{4}{3} \pi a^{3}
$$

Replacing the value of $\sigma$ and $\mathrm{v}$ in Equation (6), and calculating the volume of dust cloud $V$

$$
V=\frac{4 F D^{2} a}{3 Q_{v} B(v, T)}
$$

The density of dust grain is given by

$$
\rho=\frac{M}{V}
$$


where $M$ is the total mass of $N$ grains and $V$ be the volume. Now replacing the volume, we get the value of dust mass

$$
M=\frac{4 F D^{2} a \rho}{3 Q_{v} B(v, T)}
$$

The Equation (11) gives the values of dust mass constants [7] and [8].

Where,

$a=$ average grain size $0.1 \mu \mathrm{m}$,

$\rho=$ density of dust grain, here assumed $3000 \mathrm{~kg} \cdot \mathrm{m}^{-3}$,

$Q_{r}=$ emissivity 0.001 for $100 \mu \mathrm{m}$ and 0.0046 for $60 \mu \mathrm{m}$,

$F=f \times 1 \mathrm{MJy} / \mathrm{Str} \times 5.288 \times 10^{-9}$ where $1 \mathrm{MJy} / \mathrm{Str}=10^{-20} \mathrm{Kg} \cdot \mathrm{s}^{-2}$ and $f=$ flux density taken from IRAS map.

\subsection{Jeans Criteria}

In the gas cloud the process of star formation begins when the gas cloud is started to collapse due to its own gravity. If the mass of cloud is enough high to its potential energy exceeds its kinetic energy, then the star formation begins. This provides criteria for the critical mass. The calculation of critical mass was performed by Sir James Jeans in 1902 [9]. The jeans mass is given by

$$
M_{J}=C\left(\frac{k T}{\mu G}\right)^{3 / 2} \frac{1}{\sqrt{\rho}}
$$

where $C=1$ as suggested by [Fundamental Astronomy],

$K=$ Boltzmann's constant,

$\rho=$ Density of dust cloud,

$\mu=$ Average molecular weight,

$G=$ Gravitational constant,

$T=$ Temperature of dust cloud.

If the dust mass $(M)$ is greater than jeans mass $\left(M_{J}\right)$ then the star formation will occur otherwise the possibility of star formation will vanish.

\section{Methodology}

\subsection{Observation}

For our study we select $5^{\circ} \times 5^{\circ}$ pixel IRAS images of flux density at 60 and 100 microns (Figure 1) from our selected target Sp1 Nebula at $(329.0,+01.9)$ galactic co-ordinate. First, we inspected our region in $5^{\circ} \times 5^{\circ}$ pixel sized images through the entire range of galactic longitude latitudes. We fixed our region of interest at galactic latitude +0.4 after reducing the size of image into $1^{\circ} \times 1^{\circ}$ pixel where one of the dense Clump of sp1 nebula is located at a distance of 1450 parsec [10]. SIMBAD (http://simbad.u-strasbg.fr) also used to find and identify the nebular structure around our region of interest. Software ALADIN v11 is used for data reduction, observation and calculation. 


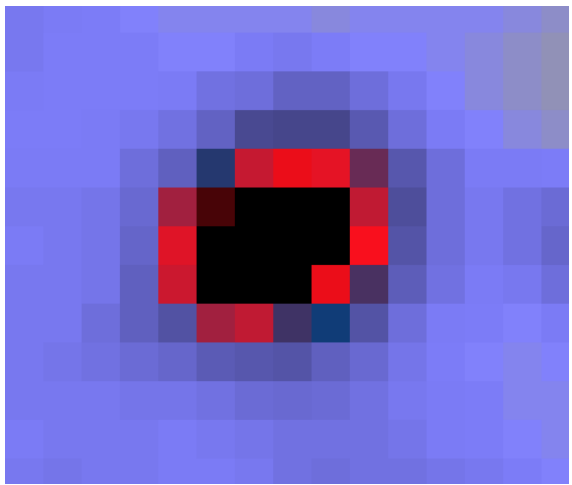

(a)

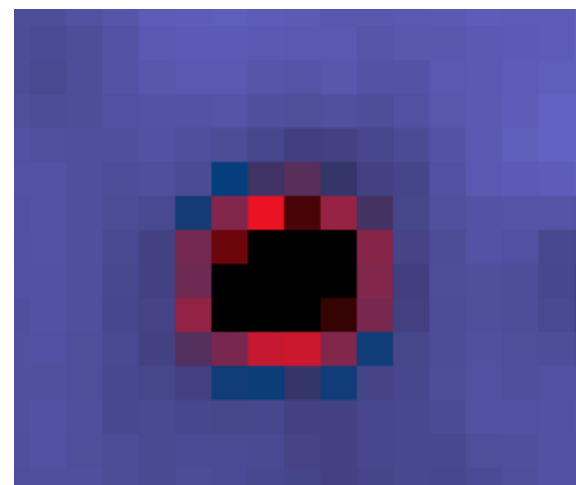

(b)

Figure 1. IRAS images of flux density. (a) IRAS image of $60 \mu \mathrm{m}$; (b) IRAS image of 100 $\mu \mathrm{m}$.

\subsection{Data}

FITS images obtained for both 100 and $60 \mu \mathrm{m}$ is studied, temperature and flux densities at a different location are taken by using ALADIN v11 software. Observing and comparing from both 60 and 100-micron images it is seen that the distribution of mass, temperature, and flux densities is found to be accumulated near the center of the structure.

For both 100 and $60 \mu \mathrm{m}$ FITS image, our region of interest was analyzed with the help of ALADIN v11 software. The flux emitted by object around the region of interest was taken for study and calculation after the background flux is filtered. The flux emitted by source object is actual peak flux and flux emitted by other sources lying nearby the region of interest but not from the region of interest is called background flux.

\subsection{Contour, Major and Minor diameter}

We have drawn the contour for both 60 and $100 \mu \mathrm{m}$ images in our region of interest with the help of ALADIN v11 software. For 60 microns we set contour level at 51,102 and 153 also, for 100 microns we fixed 108,144 and 180 contour levels. Following Figure 2 shows the contour of 60 and 100 micron images.

Also we have drawn major and minor axis in our region of interest with the help of ALADIN v11 software. The major and minor axis of big giant clump is illustrated as follows (Figure 3).

For $100 \mu \mathrm{m}$ major diameter of the structure is obtained about 10.76' while the minor diameter is obtained about 10.46 '. Also the value of major diameter for 60 $\mu \mathrm{m}$ is $12.2^{\prime}$ and $10.86^{\prime}$ for minor diameter.

\section{Result and Discussion}

There different contour levels are drawn in IRAS FITS images of $100 \mu \mathrm{m}$ and 60 $\mu \mathrm{m}$. The flux densities at different points of images are observed at each contour level. And calculate the average temperature, mass and jeans mass of selected region. 


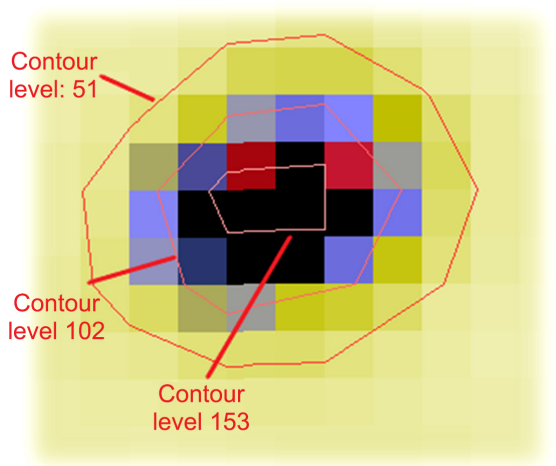

(a)

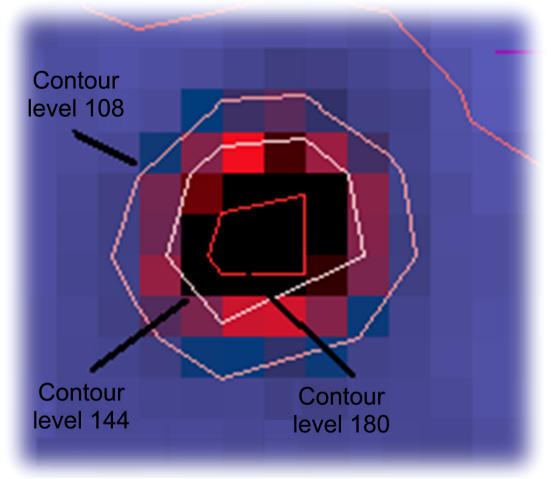

(b)

Figure 2. Obtained contour for IRAS images of $60 \mu \mathrm{m}$ and $100 \mu \mathrm{m}$. (a) Obtained Contour for IRAS image of $60 \mu \mathrm{m}$; (b) Obtained Contour for IRAS image of $100 \mu \mathrm{m}$.

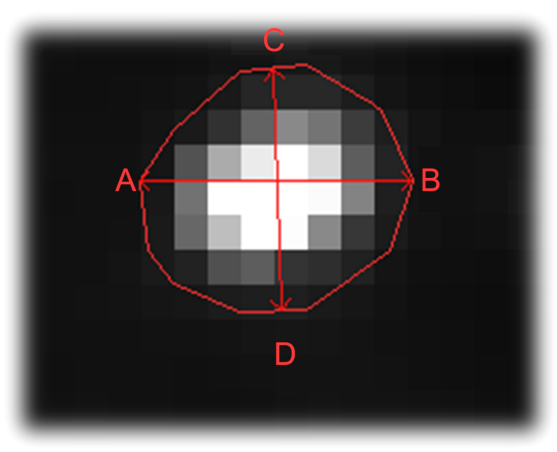

(a)

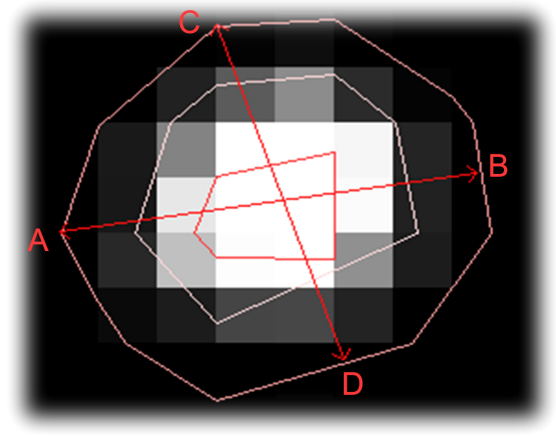

(b)

Figure 3. Major and minor diameter of $60 \mu \mathrm{m}$ and $100 \mu \mathrm{m}$ IRAS images. (a) Major and Minor diameter of $60 \mu \mathrm{m}$ IRAS image; (b) Major and Minor diameter of $100 \mu \mathrm{m}$ IRAS image.

\subsection{Variation of Flux}

\subsubsection{Distribution of flux}

The background flux is found to be centrally accumulated in both $60 \mu \mathrm{m}$ as well as $100 \mu \mathrm{m}$ image. While comparing the flux map of both 60 and $100 \mu \mathrm{m}$ (Figure 4), we have observed centrally located big hump of flux which shows the isolated property of nebular structure. Such hump gets decreased in $100 \mu \mathrm{m}$ images, which indicates the presence of dense, radiating dust like structure in central region.

\subsubsection{Variation of Flux in Major and Minor Diameter}

Study of distribution of flux over the major and minor axis shows the how flux is distributed in such region. For both $60 \mu \mathrm{m}$ and $100 \mu \mathrm{m}$ IRAS map, we observe centrally dense flux. Following graph shows the variation of flux from center of region to the outer region which follows the Gaussian distribution.

The above Gaussian distribution shows the centrally concentrated flux variation over the major and minor axis in both $60 \mu \mathrm{m}$ and $100 \mu \mathrm{m}$ IRAS images (Figure 5). 


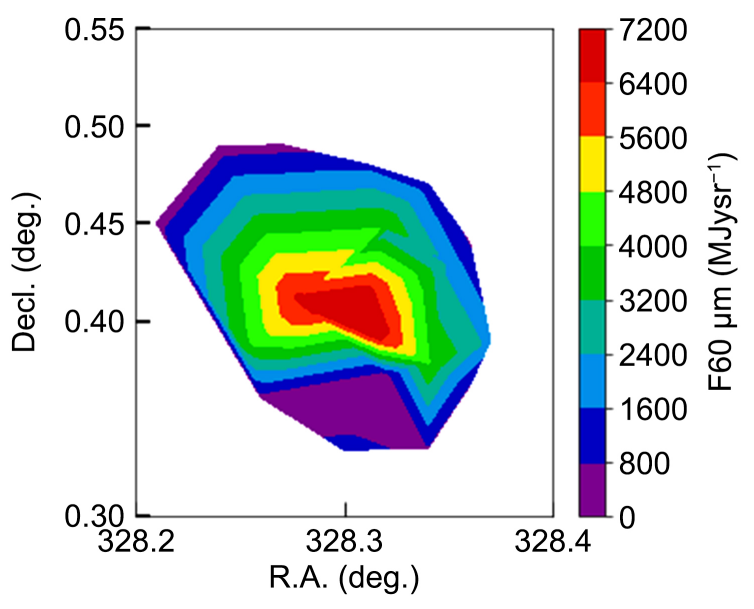

(a)

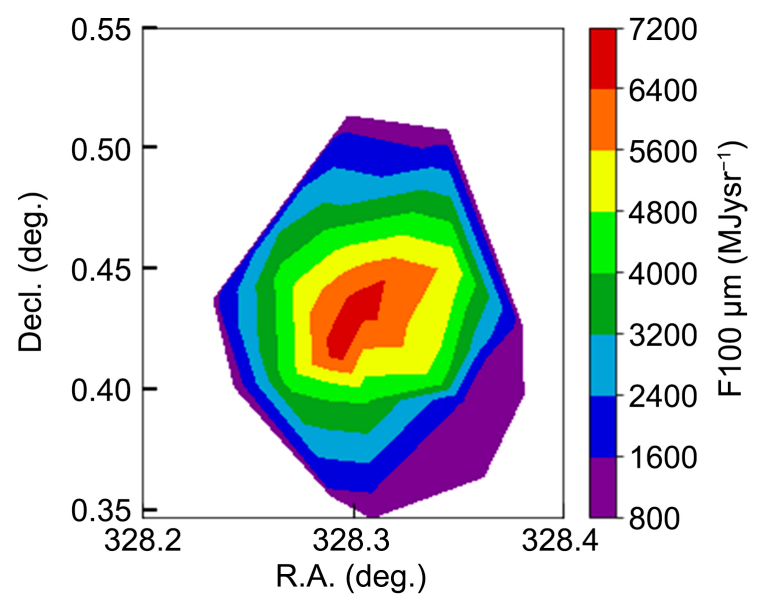

(b)

Figure 4. Distribution of flux (background corrected) over selected region. (a) Distribution of flux (background corrected) over the selected region of $60 \mu \mathrm{m}$ IRAS image; (b) Distribution of flux (background corrected) over the selected region of $100 \mu \mathrm{m}$ IRAS image.

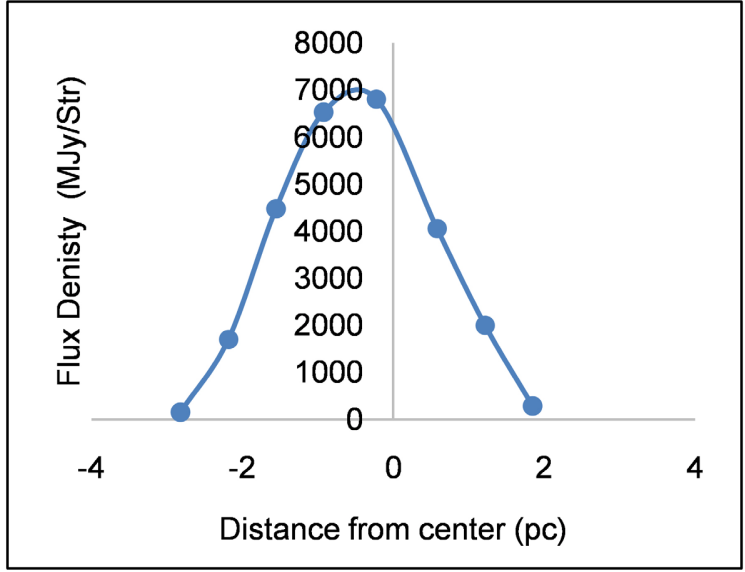

(a)

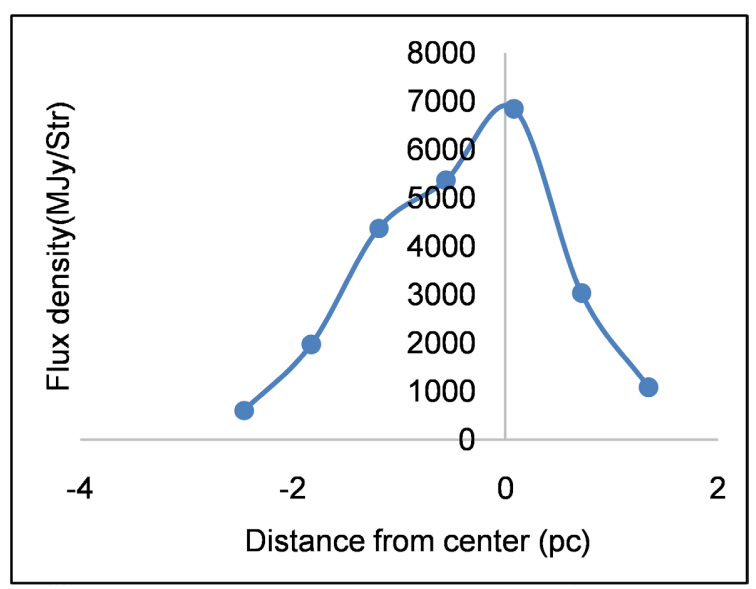

(c)

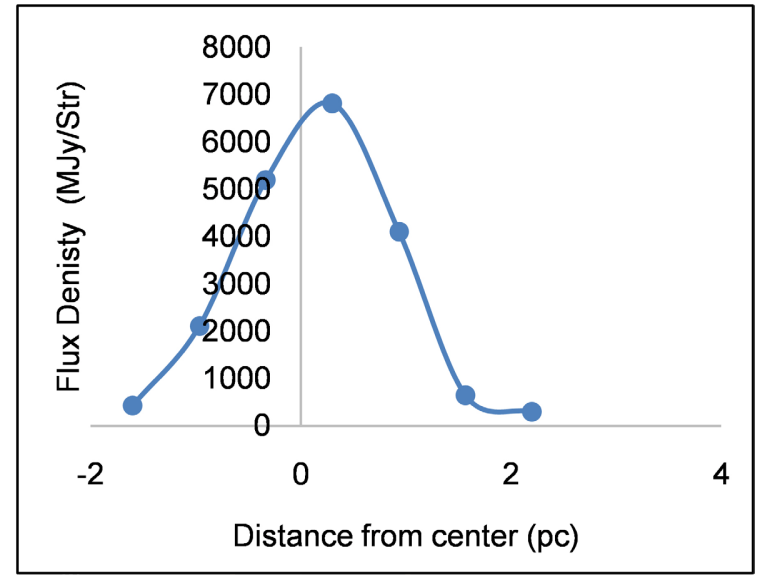

(b)

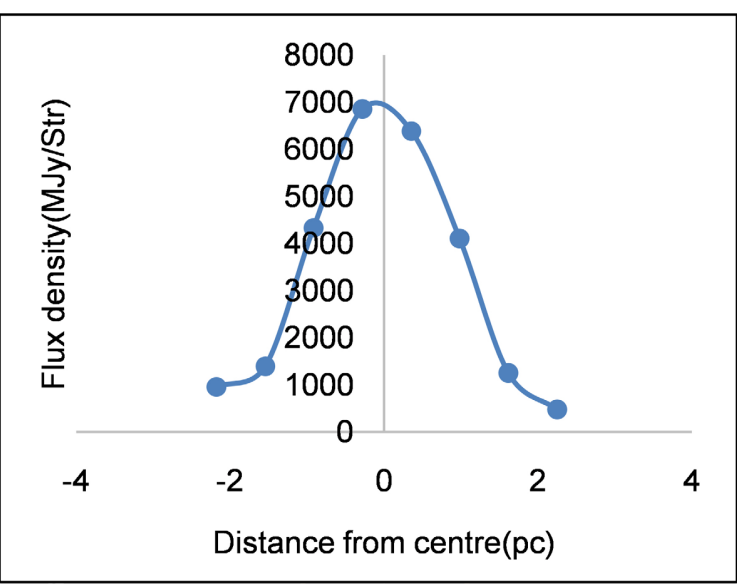

(d)

Figure 5. Flux variation along the major and minor diameter of $60 \mu \mathrm{m}$ and $100 \mu \mathrm{m}$ IRAS images. (a) Flux variation along the major diameter of $60 \mu \mathrm{m}$ IRAS image; (b) Flux variation along the minor diameter of $60 \mu \mathrm{m}$ IRAS image; (c) Flux variation along the major diameter of $100 \mu \mathrm{m}$ IRAS image; (d) Flux variation along the minor diameter of $100 \mu \mathrm{m}$ IRAS image. 


\subsection{Dust Temperature}

By observing the flux densities at $60 \mu \mathrm{m}$ and $100 \mu \mathrm{m}$ image, we calculate the ratio $\mathrm{R}$ between them. The different temperature at different contours is obtain by putting the value of $\mathrm{R}$ in equation 3 . We calculate the average temperature of all the contour level using the value $\beta=2$ [11].

Dust-gas temperature of outer region is $31.25 \mathrm{~K}$ which undergoes gradually increasing towards the core of a structure with reaching maximum temperature of $37.05 \mathrm{~K}$ (Table 1). The study shows the uniform distribution of temperature in nebular structure with hot dense core is surrounded by less cold and dense dust cloud. The average temperature of the dust cloud is $34.43 \mathrm{~K}$.

\subsection{Dust Mass}

To calculate the dust mass, the flux density of the dust cloud is measured with the help of ALADIN v11. The average value of temperature $(34.43 \mathrm{~K})$ of dust cloud is taken. The distance of the Sp1 nebula is about $1450 \mathrm{pc}$. After substituting those values in Equation (11), we get mass of dust cloud (Table 2).

\subsection{Jeans Mass}

To calculate the jeans mass, the diameter of the dust cloud is measured with the help of ALADIN v11. The average diameter of the cloud is found to be $4.47 \mathrm{pc}$. The density of the dust is calculated by assuming that the structure is spherical with diameter of $4.47 \mathrm{pc}$. The value density is obtained about $2.49385 \times 10^{-23} \mathrm{~kg}$ $\mathrm{m}^{-3}$. With the help of Equation (11) we calculated the jeans mass (Table 3).

Table 1. Temperature of nebular structure at different contour levels.

\begin{tabular}{cc}
\hline Contour Levels & Temperature (T) \\
\hline Contour Level 1 & $37.05 \mathrm{~K}$ \\
Contour Level 2 & $35 \mathrm{~K}$ \\
Contour Level 3 & $31.25 \mathrm{~K}$ \\
\hline
\end{tabular}

Table 2. Mass of dust cloud.

\begin{tabular}{cc}
\hline Mass of the Dust Cloud & Ratio to the Mass of the Sun \\
\hline $7.23718 \times 10^{28} \mathrm{KG}$ & $0.036185 \mathrm{M}_{\odot}$ \\
\hline
\end{tabular}

Table 3. The calculated value of jeans mass for the selected region.

\begin{tabular}{cc}
\hline The Jeans Mass & Ratio to the Mass of the Sun \\
\hline $5.57935 \times 10^{34} \mathrm{KG}$ & $27,896.75 \mathrm{M}_{\odot}$ \\
\hline
\end{tabular}




\section{Conclusion}

The studied nebular structure is found lighter than the mass of the sun which is about $0.036185 \mathrm{M}_{\odot}$. The average diameter of a clump is $4.47 \mathrm{pc}$. We have studied the flux density variation in both the major and minor axis of the clump. A study of flux density shows heavily concentrated condensation of dust and a Gaussian-like distribution is observed along major and minor diameters of the structure. Jeans mass of the clump structure is calculated $27,896.75 \mathrm{M}_{\odot}$, which is very high with a comparison of the actual mass of the clump and the average temperature of the region is $34.43 \mathrm{~K}$. From Gaussian distribution of flux variation over the major and minor diameter we conclude that, the system is self-isolated and less disturbed from the external factors. Structure is with radiating dense core and it is interestingly noticed that star formation in the region is not possible. But, if structure accumulates more masses than Jeans criteria (Jeans mass), the process of star formation might begin. If it is unable to collect more masses, it may turn into brown dwarf.

\section{Conflicts of Interest}

The authors declare no conflicts of interest.

\section{References}

[1] Field, G.B. and Saslaw, W.C. (1965) A Statistical Model of the Formation of Stars and Interstellar Clouds. The Astrophysical Journal, 142, 568. https://doi.org/10.1086/148318

[2] Allen, M. and Robinson, G.W. (1977) The Molecular Composition of Dense Interstellar Clouds. The Astrophysical Journal, 212, 396-415. https://doi.org/10.1086/155059

[3] Bronfman, L., Nyman, L.A. and May, J. (1996) A CS(2-1) Survey of IRAS Point Sources with Color Characteristics of Ultra-Compact HII Regions. Astronomy and Astrophysics Supplement Series, 115, 81.

[4] Schnee, S.L., Ridge, N.A., Goodman, A.A. and Li, J.G. (2005) A Complete Look at the Use of IRAS Emission Maps to Estimate Extinction and Dust Temperature. The Astrophysical Journal, 634, 442. https://doi.org/10.1086/491729

[5] Karttunen, H., Kröger, P., Oja, H., Poutanen, M. and Donner, K.J. (Eds.) (2007) Fundamental Astronomy (Vol. 4). Springer, Berlin. https://doi.org/10.1007/978-3-540-34144-4

[6] Schnee, S., Enoch, M., Noriega-Crespo, A., Sayers, J., Terebey, S., Caselli, P., Shetty, R., et al. (2009). The Dust Emissivity Spectral Index in the Starless Core TMC-1C. The Astrophysical Journal, 708, 127. https://doi.org/10.1088/0004-637X/708/1/127

[7] Hildebrand, R.H. (1983) The Determination of Cloud Masses and Dust Characteristics from Submillimetre Thermal Emission. Quarterly Journal of the Royal Astronomical Society, 24, 267-282.

[8] Young, K., Phillips, T.G. and Knapp, G.R. (1993) Circumstellar Shells Resolved in IRAS Survey Data. II-Analysis. The Astrophysical Journal, 409, 725-738. https://doi.org/10.1086/172702

[9] Thapa, A.S., Paudel, M.S. and Pant, B. (2019) An Infrared Survey of Isolated Nebu- 
lar Structures at Galactic Latitudes $16.98^{\circ} \& 1.98^{\circ}$ in ARAS Map. Journal of Nepal Physical Society, 5, 74-84. https://doi.org/10.3126/jnphyssoc.v5i1.26934

[10] Bensby, T. and Lundström, I. (2001) The Distance Scale of Planetary Nebulae. Astronomy \& Astrophysics, 374, 599-614. https://doi.org/10.1051/0004-6361:20010705

[11] Dupac, X., Bernard, J.P., Boudet, N., Giard, M., Lamarre, J.M., Mény, C. and Torre, J.P. (2003) Inverse Temperature Dependence of the Dust Submillimeter Spectral Index. Astronomy \& Astrophysics, 404, L11-L15.

https://doi.org/10.1051/0004-6361:20030575 